\title{
Usefulness of lung ultrasound B-lines in connective tissue disease-associated interstitial lung disease: a literature review
}

\author{
YuKai Wang ${ }^{1,2,3^{*}+}$, Luna Gargani ${ }^{4^{*}+}$, Tatiana Barskova ${ }^{1,2}$, Dan E. Furst ${ }^{1,2,5}$ and Marco Matucci Cerinic ${ }^{1,2}$
}

\begin{abstract}
Interstitial lung disease (ILD) is a major pulmonary manifestation of connective tissue disease (CTD), leading to significant morbidity and mortality. Chest high-resolution computed tomography (HRCT) is presently considered the diagnostic gold standard for pulmonary fibrosis diagnosis and quantification in the clinical arena. However, not negligible doses of ionizing radiation limit the use of HRCT, especially for serial follow-up in younger female patients. In the past decade, lung ultrasound (LUS) has been proposed to assess ILD by detecting and quantifying sonographic B-lines. Previous studies demonstrate that B-lines have a good diagnostic accuracy, especially high sensitivity, and correlate well with HRCT findings, suggesting LUS as a novel, non-invasive, and non-ionizing imaging method to be used in patients with CTD-ILD. Although preliminary data are promising, challenges and controversies still remain. For example, the mechanisms of B-line generation are not fully understood; the diagnostic accuracy and performance characteristics of LUS partially depend on the scanning scheme and scoring system used; and up-to-date B-lines cannot discriminate the early cellular inflammation from the chronic fibrotic phase in CTD-ILD. Therefore it is important for clinicians to understand the strengths and limitations of LUS in CTD-ILD patients, to maximize its value.
\end{abstract}

Keywords: Lung ultrasound, B-lines, Pleural irregularity, High-resolution computed tomography, Connective tissue diseases, Interstitial lung disease, Systemic sclerosis, Rheumatoid arthritis, Sjögren's syndrome, Anti-synthetase syndrome

\section{Background}

The lung parenchyma has always been considered a "forbidden zone" for ultrasound (US), because air is not a favourable medium for transmission of US waves. As a consequence, thoracic US was originally limited to the study of superficial pleural conditions, such as tumours, effusions, and to guide invasive procedures [1]. In the last decade, the so-called B-lines have been recognized as the sonographic sign of the pulmonary interstitial syndrome [2]. This sign is thought, at present, to reflect partial deaeration of the lung, which may be due to fluid accumulation [1-4] or deposition of collagen tissue [1, 2]. Studies in patients with diffuse parenchymal lung diseases have

\footnotetext{
* Correspondence: stzxyywyk@126.com; gargani@ifc.cnr.it

'Equal contributors

${ }^{1}$ Department of Experimental and Clinical Medicine, University of Florence,

Florence, Italy

${ }^{4}$ Institute of Clinical Physiology, National Research Council, Via Moruzzi 1,

56124 Pisa, Italy

Full list of author information is available at the end of the article
}

attempted to highlight the usefulness of LUS as a complementary modality to traditional radiologic imaging $[5,6]$. In this paper, we review all data about the usefulness of B-lines to CTD-ILD, underlining the strengths and limitations.

\section{What are B-lines?}

In radiologic imaging, the term "artefact" describes any part of an image which does not accurately represent the anatomic structures present within the subject being evaluated [7]. B-lines are defined as discrete laser-like vertical hyperechoic reverberation artefacts that arise from the pleural line, extend to the bottom of the screen without fading, and move synchronously with respiration [8]. B-lines are visible when the lung parenchyma air content is partially decreased and/or the interstitial space is volumetrically expanded, such as in pulmonary oedema of various aetiologies and interstitial lung disease [9-11]. It is important to underline that B-lines 
cannot be clearly correlated to a specific anatomical structure, but rather they are correlated to the changes in the physical properties of the lung [10]. Soldati et al. [9-11] hypothesized that the mechanism underlying B-line formation is reverberation coherent with topologic and pathologic variations of the lung interstitium. Although the results of these first experiments are promising, the biophysics and exact genesis of B-lines are not yet fully elucidated.

\section{B-lines in connective tissue disease-associated interstitial lung disease}

Pulmonary involvement is a significant cause of CTDrelated morbidity and mortality [12, 13]. Interstitial lung disease is a frequent parenchymal manifestation of CTDs. Although the pathogenesis of CTD-ILD is varied and not yet fully understood, early detection and therapy may improve the prognosis [14]. To date high-resolution computed tomography (HRCT) is the gold standard to diagnose CTD-ILD [15, 16]. Unfortunately, HRCT cannot be repeated very often because it has a high cost and is associated with high radiation exposure $[17,18]$. Furthermore, although pulmonary function tests (PFTs) are valuable in screening and following-up for CTD-ILD, they are not always impaired in early stages and may not reflect the degree of fibrosis [19]. Lung biopsy is a powerful tool to establish a definite histopathologic diagnosis of ILD, but the invasiveness and the possibility of sampling errors limit its clinical application [20]. Since LUS is a non-invasive and nonionizing modality, rheumatologists and internists attempted to use it to assess the presence of CTD-ILD. Preliminary data showed that the number of B-lines had a good correlation with the HRCT fibrosis pattern, and good diagnostic accuracy, especially sensitivity [21], expanding the armamentarium for diagnosis and follow-up of CTD-ILD.

\section{B-lines in systemic sclerosis-associated interstitial lung disease}

Systemic sclerosis (SSc) is most often associated with ILD, with up to $90 \%$ of patients exhibiting evidence of
ILD on HRCT [22]. Accordingly, the majority of studies about B-lines in CTD-ILD focused on SSc. The use of B-lines has been partially validated in SSc-ILD. Different studies, as discussed in the following, established the use of B-lines in over 400 patients with a wide range of disease duration, type, and severity.

A scoring system for LUS is needed to examine construct and criterion validity. In the past decade, some scoring methods have been developed and used to quantify the disease. In the first study about B-lines in SScILD, a B-line score was calculated in $33 \mathrm{SSc}$ patients by summing the total number of B-lines on the anterior and posterior chest. A total of 72 scanning sites were analysed. The examination was considered positive when the B-line sum in all scanning sites was $>10$ [23]. In another study, a positive examination was defined either when $\geq 3$ B-lines were present in at least two adjacent scanning sites or when a total of $>5$ B-lines was recorded [24]. Gutierrez et al. [25] compared two different LUS methods to assess the CTD-ILD in a single cohort of patients (Table 1). The number of B-lines in 50 scanning sites (comprehensive assessment) and 14 scanning sites (simplified assessment) located among the bilateral anterior, medial, and posterior chest was counted. For the comprehensive assessment a B-line semi-quantitative score was defined by $0=$ normal $(<10$ B-lines $), 1=$ mild (11-20 B-lines), $2=$ moderate $(21-50$ B-lines), and $3=$ marked ( $>50$ B-lines), whereas for the simplified assessment the semi-quantitative score was $0=$ normal $(<5$ B-lines), 1 $=$ mild (6-15 B-lines), $2=$ moderate (16-30 B-lines), and 3 $=$ marked $(>30$ B-lines). The study found a significant cor relation between the two scoring systems $(p=0.0001)$, with $\kappa$ values for the inter-observer simplified LUS assessment in the range of $0.769-0.885$, and concordance correlation coefficient values for the intra-observer reliability from 0.856 to 0.955 . The simplified method required less time than the comprehensive examination (mean $8.6 \pm 1.4 \mathrm{mi}-$ nutes vs $23.3 \pm 4.5$ minutes, $p<0.0001)$. Recently, fewer scanning sites (only 10) were evaluated based on the

Table 1 Four different LUS methods to assess B-lines in SSC patients

\begin{tabular}{|c|c|c|c|c|c|c|c|c|c|}
\hline & \multirow[t]{2}{*}{ Anatomical line } & \multicolumn{2}{|c|}{ LUS by Gargani et al. [23] } & \multicolumn{2}{|c|}{ Comprehensive LUS [25] } & \multicolumn{2}{|c|}{ Simplified LUS [25] } & \multicolumn{2}{|c|}{ Modified LUS [26] } \\
\hline & & Right & Left & Right & Left & Right & Left & Right & Left \\
\hline \multirow[t]{2}{*}{ Anterior } & Parasternal & 2nd-5th ICS & 2nd-4th ICS & 2nd-5th ICS & 2nd-4th ICS & 2nd ICS & 2nd ICS & & \\
\hline & Mid-clavicular & 2nd-5th ICS & 2nd-4th ICS & 2nd-5th ICS & 2nd-4th ICS & 4th ICS & 4th ICS & 4th ICS & 4th ICS \\
\hline \multirow[t]{3}{*}{ Lateral } & Anterior axillary & 2nd-5th ICS & 2nd-4th ICS & 2nd-5th ICS & 2nd-4th ICS & 4th ICS & 4th ICS & 4th ICS & 4th ICS \\
\hline & Mid-axillary & 2nd-5th ICS & 2nd-4th ICS & 2nd-5th ICS & 2nd-4th ICS & 4th ICS & 4th ICS & 4th ICS & 4th ICS \\
\hline & Posterior axillary & 2nd-10th ICS & 2nd-10th ICS & 7th-8th ICS & 7th-8th ICS & 8th ICS & 8th ICS & 8th ICS & 8th ICS \\
\hline \multirow[t]{2}{*}{ Posterior } & Sub-scapular & 7th-10th ICS & 7th-10th ICS & 7th-8th ICS & 7th-8th ICS & 8th ICS & 8th ICS & 8th ICS & 8th ICS \\
\hline & Paravertebral & 2nd-10th ICS & 2nd-10th ICS & 2nd-8th ICS & 2nd-8th ICS & 8th ICS & 8th ICS & & \\
\hline \multicolumn{2}{|c|}{ Total scanning sites } & $72 \mathrm{ScS}$ & & $50 \mathrm{ScS}$ & & $14 \mathrm{ScS}$ & & $10 \mathrm{ScS}$ & \\
\hline
\end{tabular}

LUS lung ultrasound, SSC systemic sclerosis, ICS inter-costal space, SCS scanning sites 
prevalence of B-line distribution. Results found that this modified scoring system had a good correlation with HRCT (correlation coefficient $=0.695, p<0.001$ ), good intraobserver reliability ( $\mathrm{K}$ value $=0.838$ ), and was the least time consuming (mean 5.4 \pm 1.8 minutes) [26] (Table 1). However, considering that ILD is usually diffuse, a more comprehensive and careful assessment may be more accurate, especially for screening purposes.

Face validity of LUS has been generally accepted as exemplified by its use when examining SSc-ILD. Construct validity requires correlating LUS with other measures of the same type or reflecting the same pathology, as well as discriminating B-lines for measures of negatively associated aspects of disease. These are called convergent and divergent correlations. These studies are often done using HRCT, thus examining criterion validity at the same time (assuming HRCT is a gold standard for ILD in SSc). Several HRCT scoring methods have been used to characterize and quantify the disease; the Warrick score, a semi-quantitative assessment combining severity and extent of disease, has been applied preferentially [27].

In the study by Gargani et al. [23], the presence of Blines was observed in $51 \%$ of SSc patients, with significantly higher values in the diffuse than in the limited form (73 \pm 66 vs $21 \pm 35 ; p<0.05$ ). A statistically significant positive linear correlation was found between $\mathrm{B}$-lines and the Warrick score $(r=0.72 ; p<0.001)$, and between Blines and values of diffusing capacity for carbon monoxide (DLCO) $(r=-0.6 ; p<0.05)$. The intra-observer and interobserver variability of B-line assessment were derived from a previous study by the same group as $5.1 \%$ and $7.4 \%$ respectively [4].

Tardella et al. [28] also reported a significant linear correlation between the number of B-lines and HRCT score $(p<0.001$; correlation coefficient $\rho=0.875)$ and between B-lines and DLCO $(p=0.014)$ in 34 CTD patients (including $26 \mathrm{SSc}$ patients). Inter-observer assessment showed very good agreement (weighted $\mathrm{k}$ value between 0.846 and 0.969 , and overall agreement between $92 \%$ and $97 \%$ ). Another study of 58 consecutive SSc patients (including 32 patients with very early SSc) showed a concordance rate of 0.83 between B-lines and HRCT for the assessment of ILD [29]. LUS diagnostic sensitivity and specificity were $100 \%$ and $55 \%$ respectively, and the negative predictive value (NPV) and positive predictive value (PPV) were $100 \%$ and $78 \%$ (with a higher cutoff point of $\geq 20$ total B-lines, sensitivity was instead $83 \%$ and specificity was 96\%). The authors also found that patients with ground glass opacity (GGO) by HRCT had a higher total B-line score than those without GGO. Receiver operating characteristic curve (ROC) analysis confirmed the analytical relationship between number of B-lines and the presence of ILD at HRCT (AUC $=0.94$, 95\% CI $0.89-0.99, p<0.0001)$. Given the very high sensitivity and negative predictive value, this study proposes B-lines as a screening tool of ILD in SSc patients, to guide further investigation with HRCT. Buda et al. [30] also observed numerous B-lines with a "white lung" pattern to be associated with GGO $(p<0.0001)$, and the sensitivity and specificity were respectively $95 \%$ and $99 \%$. In another study comparing $25 \mathrm{SSc}$ patients to 40 healthy controls, all SSc patients with CT signs of ILD $(44 \%)$ showed B-lines versus only $7 \%$ of healthy controls $(p<0.001)$ [31]. Pleural irregularities (PI, defined as the loss of the normal hyperechoic linear pleural contour plus thickening) were also described in this study, although their anatomic correlations and validity are still debated $[1,32]$. LUS findings matched the findings on HRCT. Patients with ILD had a higher number of Blines and higher pleural scores compared with those without radiographic ILD. A similar outcome was observed in a small heterogeneous group of CTD patients (including 25 RA patients, 14 SSc patients, and 6 SLE patients). B-lines were detected in $100 \%$ and $12 \%$ of patients with or without HRCT-defined ILD, respectively [33]. Subpleural nodes and pleural thickness $>3 \mathrm{~mm}$ were observed in 55\% and 95\% of ILD patients compared to $17 \%$ and $12.5 \%$ of patients without ILD. In another study of 16 SSc and 21 anti-synthetase syndrome (ASS) patients, PI again showed a high accuracy for detecting radiological ILD [32]. Another study in 175 SSc patients reported that pleural line thickness and subpleural nodules had a good concordance with HRCT patterns indicating pulmonary fibrosis severity, and were able to detect signs of initial pulmonary fibrosis prior to the onset of respiratory symptoms [34].

Several papers have highlighted the correlation between LUS signs with some clinical features. The relationships between B-lines, PFTs, and clinical variables were evaluated in 39 SSc patients [24]. This study confirmed previous data from Gargani et al. [23], showing that the B-line score had a negative correlation with DLCO $(r=-0.63, p$ $<0.0001)$. The number of B-lines increased as capillaroscopic damage in the fingers increased $(p<0.01)$. In addition, the B-line score was significantly higher in patients with diffuse cutaneous SSc (dcSSc) than in those with limited cutaneous SSc (lcSSc) $(p<0.05)$, and in patients with digital ulcer history than in those without digital ulcer history $(p<0.01)$. The Medsger scleroderma disease severity scale also had a significant correlation with B-lines $(r=0.80, p<0.01)$. No significant association was observed with disease duration, mRSS, or European Scleroderma Study Group activity index.

\section{B-lines in rheumatoid arthritis-associated interstitial lung disease}

There are two studies examining LUS in RA patients $[35,36]$. A prospective study of 64 RA outpatients 
without clinical pulmonary symptoms revealed that $28 \%$ of patients had B-lines or pleural nodules [35]. In $89 \%$ of LUS-positive patients, HRCT scans showed signs of ILD. This established criterion validity for LUS with the "usual" assumption that HRCT represents the gold standard. LUS also showed sporadic abnormalities in $7 \%$ of the healthy controls. Agreement between LUS and HRCT yielded a sensitivity of $97.1 \%$ and a specificity of $97.3 \%$. The predefined criteria yielded a PPV of $94.3 \%$ and NPV of $98.6 \%$. These encouraging results will need to be corroborated in larger studies.

Another study has compared two different US devices to detect B-lines in a small cohort of RA patients. Both standard (using a $2-5 \mathrm{MHz}$ convex probe) and pocketsize US (PS-US, using a 1.7-3.8 $\mathrm{MHz}$ phased array transducer) devices were used to examine lungs characterized by radiological ILD in 39 RA patients [36]. A Bline score $>10$ identified a positive examination. The study found that sensitivity and specificity of standard LUS and PS-LUS vs HRCT were $92 \%$ and $56 \%$, and $89 \%$ and $50 \%$, respectively. The $\mathrm{k}$ coefficient between the two methods was 0.78 , indicating that PS-US devices can provide a diagnostic accuracy similar to higher-level devices.

These data may help define the utility of LUS in RA, but other crucial aspects of this device have not yet been proven valid in RA, including its reproducibility, reliability, and applicability to a wide range of patients. Responsiveness and discrimination are unknown. Hopefully, much of the work done in SSc can be applied here, but caution is justified.

\section{B-lines in Sjögren's syndrome-associated interstitial lung disease}

More recently, it has been demonstrated that B-lines are well correlated to the HRCT sign of pulmonary fibrosis in Sjögren's syndrome (SS) patients [37]. Thirteen SS patients were evaluated by LUS and chest HRCT, independently performed within 6 months. Blines were evaluated on eight thoracic zones. A zone was considered positive if at least three B-lines were identified in a single ICS. LUS showed a sensitivity of 1 (95\% CI $0.398-1.0)$, a specificity of 0.89 (95\% CI $0.518-0.997)$, and a positive probability reason of 9 (95\% CI 7.1-11.3) to detect ILD. LUS had good correlation with HRCT $(r=0.84, p<0.001)$ and high accuracy to diagnose ILD (AUC $=-0.94,95 \%$ CI $0.81-1.0, p=0.014)$. Although the data are promising, the positive evidence relies on a very small number of patients. Another limitation is the long time frame between LUS and HRCT, with potential bias linked to changes in lung parenchyma over time.

\section{B-lines in anti-synthetase syndrome-associated interstitial lung disease}

The correlation of B-lines with HRCT was studied in 22 ASS patients [38]. B-lines were analysed semi-quantitatively (a maximum of B-lines, calculated as the percentage of positive sonographic points, divided by the number of sonographic points studied per patient) and were most often found in the lower posterior and upper anterior areas. The $\mathrm{k}$ values for intra-observer and inter-observer reliability were 0.83 and 0.76 , respectively. The median HRCT Warrick score was 15 (Q1-Q3 13-22), with GGO affecting the largest number of segments (median 10 (Q1-Q3 6-12)), followed by irregular pleural margins (median 6 (Q1-Q3 4110)), and septal/subpleural lines (median 6 (Q1-Q3 0-10)). No significant correlation was found between the percentage of B-lines and the overall Warrick score. When correlation with the different components of Warrick's score was analysed, only the number of HRCT segments showing GGO was related to the percentage of B-lines $(\rho=0.5, p=0.02)$. In a subsequent study from the same group on patients with both SSc and ASS, PI showed a better performance than B-lines to detect ILD [32]. These results are partially not consistent with previous studies; a possible explanation being the different scoring systems. Further research is needed to better understand the role of LUS in ASS, and especially to evaluate the role of PI in these conditions.

All of the included studies are depicted in Table 2. Different HRCT and LUS patterns of absent, moderate, and severe fibrotic involvement are shown in Fig. 1.

\section{Limitations}

The studies outlined showed promising results in selected diseases and circumstances. LUS is almost fully validated in SSc (lacking only discrimination) and SS (very small number patients), and is incompletely validated in RA and ASS. Complete validation is lacking in all of these diseases, making the use of LUS somewhat preliminary, despite being very promising and attractive.

B-lines can have various aetiologies (e.g. interstitial oedema, interstitial fibrosis) $[8,39,40]$, and differentiating them in clinical practice can sometimes be difficult. In particular, in CTD-ILD B-lines alone cannot differentiate the early inflammatory phase from the chronic fibrotic phase [41, 42], which could potentially lead to some misclassification bias, although this differentiation is also often not so easy by HRCT. The role of pleural abnormalities, which seems promising in completing the information provided by B-lines, warrants more specific studies, and it is debatable whether measuring the sonographic pleural line could be meaningful in normal and pathologic conditions.

Up to now, no method to objectively score B-lines has been provided (although computer algorithms are under 


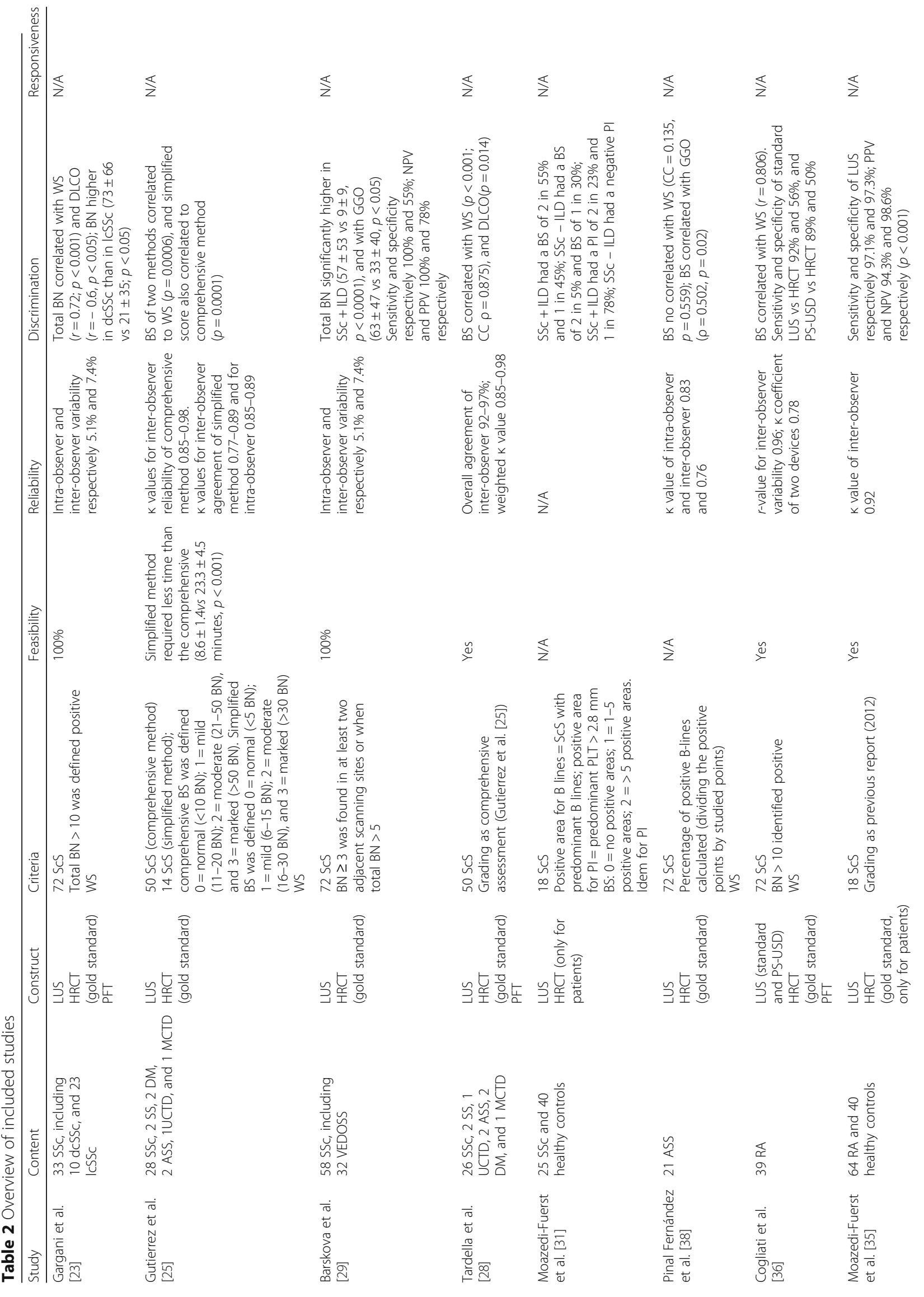




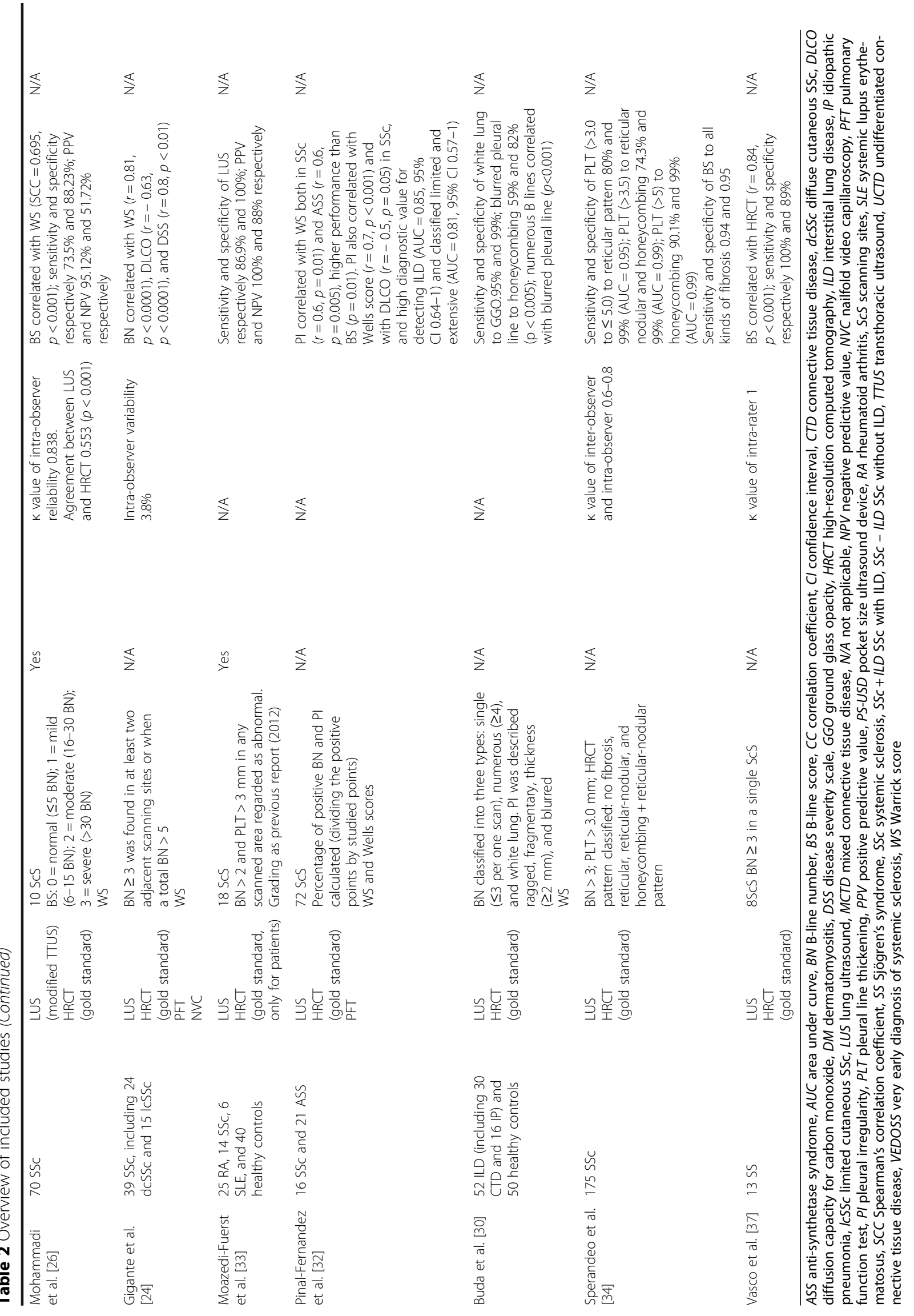



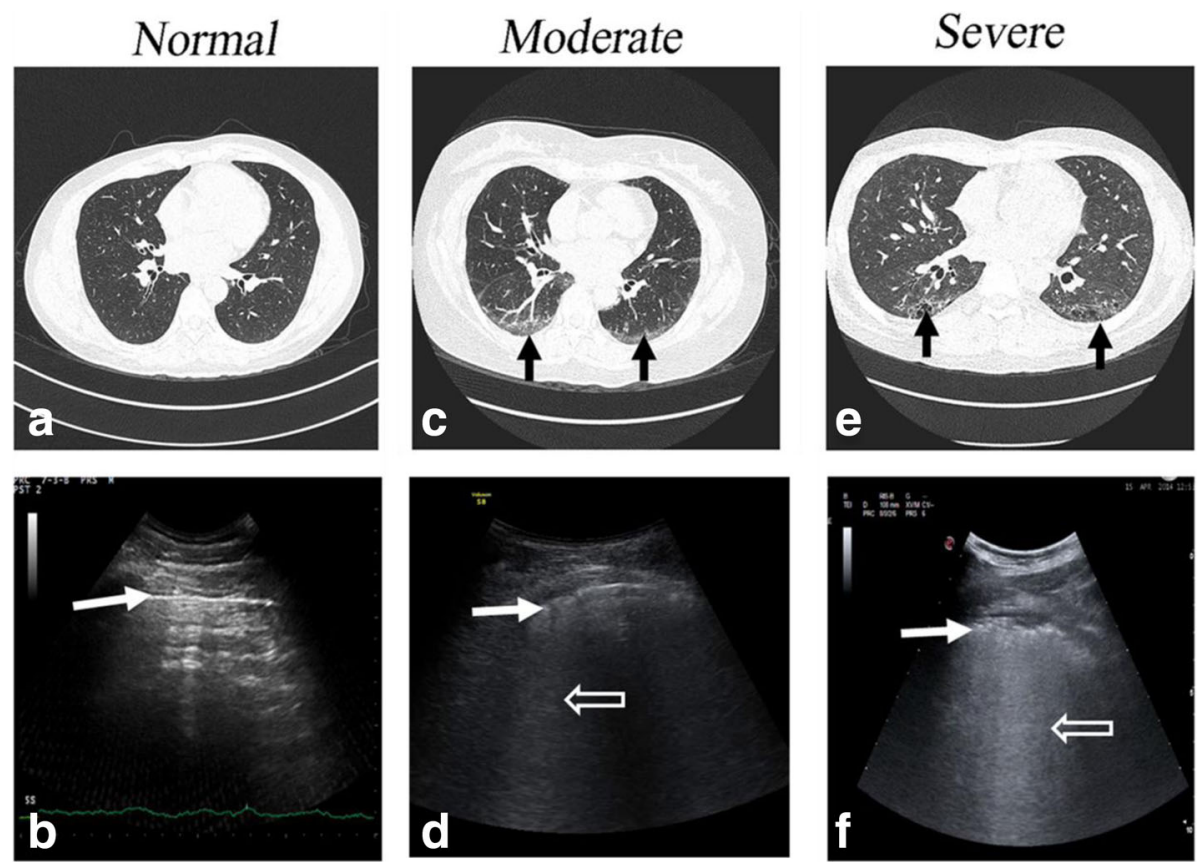

Fig. 1 Different HRCT and LUS patterns. a HRCT: normal. b LUS: normal pleural line (white arrow). No B-lines visible. c HRCT: ground-glass opacity (black arrow). d LUS: blurred and irregular pleural line (white arrow) and multiple B-lines (white empty arrow). e HRCT: honey-combing (black arrow). f LUS: blurred and irregular pleural line (white arrow) and multiple B-lines ("white lung", white empty arrow). HRCT high-resolution computed tomography, LUS lung ultrasound

investigation) $[43,44]$. In the future, this area should be a priority for research.

Furthermore, the majority of studies focused on Blines as a diagnostic tool in ILD. No data are yet available on B-lines for follow-up in rheumatic disease patients, nor on the accuracy of this method to assess the eventual response to therapy (only two case reports are available) $[45,46]$, nor on the correct timing of LUS for diagnosis and follow-up. All studies up to now have included rather small populations from a single centre.

Finally, no data are available on large normal populations to confirm the cut-off points and PPV and NPV for this technique in SSc, RA, and other CTDs [47-50].

\section{Conclusions}

LUS is an attractive and promising technique, which may become an important clinical tool to be integrated with HRCT and PFT in the screening and evaluation of ILD. To date, B-lines are waiting to be validated fully in CTD, and the role and meaning of sonographic pleural irregularities must be more clearly elucidated.

\section{Abbreviations}

ASS: Anti-synthetase syndrome; CTD-ILD: Connective tissue diseaseassociated interstitial lung disease; dcSSc: Diffuse cutaneous systemic sclerosis; DLCO: Diffusing capacity for carbon monoxide; GGO: Ground glass opacity; HRCT: High-resolution computed tomography; ICSSC: Limited cutaneous systemic sclerosis; LUS: Lung ultrasound; NPV: Negative predictive value; PFT: Pulmonary function test; PI: Pleural irregularities; PPV: Positive predictive value; PS-US: Pocket-size ultrasound; RA: Rheumatoid arthritis; ROC: Receiver operating curve; SLE: Systemic lupus erythematosus; SSc: Systemic sclerosis; US: Ultrasound

\section{Acknowledgements}

Not applicable.

Funding

Not applicable.

Availability of data and materials Not applicable.

Authors' contributions

$Y W, L G, T B, D E F$, and $M M C$ participated in the literature review, figure design, and writing of the review. All authors read and approved the final manuscript.

Ethics approval and consent to participate Not applicable.

\section{Consent for publication}

Not applicable.

\section{Competing interests}

$L G$ received speaker honoraria from GE Healthcare and Glaxo-Smith-Kline. The remaining authors declare that they have no competing interests.

\section{Publisher's Note}

Springer Nature remains neutral with regard to jurisdictional claims in published maps and institutional affiliations. 


\section{Author details}

'Department of Experimental and Clinical Medicine, University of Florence, Florence, Italy. ${ }^{2}$ Department of Geriatric Medicine, Division of Rheumatology AOUC, University of Florence, Florence, Italy. ${ }^{3}$ Department of Rheumatology and Immunology, Shantou Central Hospital, No 114 Waima Road, Shantou 515041, Guangdong, China. ${ }^{4}$ Institute of Clinical Physiology, National Research Council, Via Moruzzi 1, 56124 Pisa, Italy. ${ }^{5}$ Division of Rheumatology, Department of Medicine, University of California at Los Angeles, Los Angeles, CA, USA.

\section{Published online: 18 September 2017}

\section{References}

1. Gargani L, Volpicelli G. How I do it: lung ultrasound. Cardiovasc Ultrasound. 2014;12:25.

2. Lichtenstein D, Meziere G, Biderman P, Gepner A, Barre O. The comet-tail artifact. An ultrasound sign of alveolar-interstitial syndrome. Am J Respir Crit Care Med. 1997;156:1640-6.

3. Agricola E, Bove T, Oppizzi M, Marino G, Zangrillo A, Margonato A, Picano E. "Ultrasound comet-tail images": a marker of pulmonary edema: a comparative study with wedge pressure and extravascular lung water. Chest. 2005;127:1690-5.

4. Jambrik Z, Monti S, Coppola V, Agricola E, Mottola G, Miniati M, Picano E. Usefulness of ultrasound lung comets as a nonradiologic sign of extravascular lung water. Am J Cardiol. 2004;93:1265-70.

5. Reissig A, Kroegel C. Transthoracic sonography of diffuse parenchymal lung disease: the role of comet tail artifacts. J Ultrasound Med. 2003;22:173-80.

6. Vizioli L, Ciccarese F, Forti P, Chiesa AM, Giovagnoli M, Mughetti M, Zompatori $\mathrm{M}$, et al. Integrated use of lung ultrasound and chest X-ray in the detection of interstitial lung disease. Respiration. 2017:93:15-22.

7. Feldman MK, Katyal S. Blackwood MS. US artifacts. Radiographics. 2009;29: 1179-89.

8. Volpicelli G, Elbarbary M, Blaivas M, Lichtenstein DA, Mathis G, Kirkpatrick AW, Melniker L, et al. International evidence-based recommendations for point-of-care lung ultrasound. Intensive Care Med. 2012;38:577-91.

9. Soldati G, Copetti R, Sher S. Sonographic interstitial syndrome: the sound of lung water. J Ultrasound Med. 2009;28:163-74.

10. Soldati $G$, Inchingolo R, Smargiassi A, Sher S, Nenna R, Inchingolo CD, Valente S. Ex vivo lung sonography: morphologic-ultrasound relationship. Ultrasound Med Biol. 2012;38:1169-79.

11. Soldati $G$, Demi M, Inchingolo R, Smargiassi A, Demi L. On the physical basis of pulmonary sonographic interstitial syndrome. J Ultrasound Med. 2016;35: 2075-86.

12. Cappelli S, Bellando Randone S, Camiciottoli G, De Paulis A, Guiducci S, Matucci-Cerinic M. Interstitial lung disease in systemic sclerosis: where do we stand? Eur Respir Rev. 2015;24:411-9.

13. Fischer A, Chartrand S. Assessment and management of connective tissue disease-associated interstitial lung disease. Sarcoidosis Vasc Diffuse Lung Dis. 2015;32:2-21.

14. Vij R, Strek ME. Diagnosis and treatment of connective tissue diseaseassociated interstitial lung disease. Chest. 2013;143:814-24.

15. Desai SR, Veeraraghavan S, Hansell DM, Nikolakopolou A, Goh NS, Nicholson AG, Colby TV, et al. CT features of lung disease in patients with systemic sclerosis: comparison with idiopathic pulmonary fibrosis and nonspecific interstitial pneumonia. Radiology. 2004;232:560-7.

16. Ohno Y, Koyama H, Yoshikawa T, Seki S. State-of-the-art imaging of the lung for connective tissue disease (CTD). Curr Rheumatol Rep. 2015;17:69.

17. Picano E, Matucci-Cerinic M. Unnecessary radiation exposure from medical imaging in the rheumatology patient. Rheumatology. 2011;50:1537-9.

18. Picano E, Semelka R, Ravenel J, Matucci-Cerinic M. Rheumatological diseases and cancer: the hidden variable of radiation exposure. Ann Rheum Dis. 2014:73:2065-8

19. Best AC, Meng J, Lynch AM, Bozic CM, Miller D, Grunwald GK, Lynch DA. Idiopathic pulmonary fibrosis: physiologic tests, quantitative $\mathrm{CT}$ indexes, and CT visual scores as predictors of mortality. Radiology. 2008;246:935-40.

20. Hunninghake GW, Zimmerman MB, Schwartz DA, King Jr TE, Lynch J, Hegele R, Waldron J, et al. Utility of a lung biopsy for the diagnosis of idiopathic pulmonary fibrosis. Am J Respir Crit Care Med. 2001;164: 193-6.
21. Song G, Bae SC, Lee YH. Diagnostic accuracy of lung ultrasound for interstitial lung disease in patients with connective tissue diseases: a metaanalysis. Clin Exp Rheumatol. 2016;34:11-6.

22. Herzog EL, Mathur A, Tager AM, Feghali-Bostwick C, Schneider F, Varga J. Review: interstitial lung disease associated with systemic sclerosis and idiopathic pulmonary fibrosis: how similar and distinct? Arthritis Rheumatol. 2014;66:1967-78.

23. Gargani L, Doveri M, D'Errico L, Frassi F, Bazzichi ML, Delle Sedie A, Scali MC, et al. Ultrasound lung comets in systemic sclerosis: a chest sonography hallmark of pulmonary interstitial fibrosis. Rheumatology (Oxford). 2009;48: 1382-7.

24. Gigante A, Rossi Fanelli F, Lucci S, Barilaro G, Quarta S, Barbano B, Giovannetti A, et al. Lung ultrasound in systemic sclerosis: correlation with high-resolution computed tomography, pulmonary function tests and clinical variables of disease. Intern Emerg Med. 2016;11:213-7.

25. Gutierrez M, Salaffi F, Carotti M, Tardella M, Pineda C, Bertolazzi C,

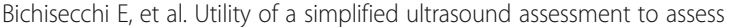
interstitial pulmonary fibrosis in connective tissue disorders-preliminary results. Arthritis Res Ther. 2011;13:R134.

26. Mohammadi A, Oshnoei S, Ghasemi-rad M. Comparison of a new, modified lung ultrasonography technique with high-resolution $C T$ in the diagnosis of the alveolo-interstitial syndrome of systemic scleroderma. Med Ultrason. 2014;16:27-31.

27. Warrick JH, Bhalla M, Schabel SI, Silver RM. High resolution computed tomography in early scleroderma lung disease. J Rheumatol. 1991;18:1520-8.

28. Tardella M, Gutierrez M, Salaffi F, Carotti M, Ariani A, Bertolazzi C, Filippucci $E$, et al. Ultrasound in the assessment of pulmonary fibrosis in connective tissue disorders: correlation with high-resolution computed tomography. J Rheumatol. 2012;39:1641-7.

29. Barskova T, Gargani L, Guiducci S, Randone SB, Bruni C, Carnesecchi G, Conforti $\mathrm{ML}$, et al. Lung ultrasound for the screening of interstitial lung disease in very early systemic sclerosis. Ann Rheum Dis. 2013;72:390-5.

30. Buda N, Piskunowicz M, Porzezinska M, Kosiak W, Zdrojewski Z. Lung ultrasonography in the evaluation of interstitial lung disease in systemic connective tissue diseases: criteria and severity of pulmonary fibrosis_analysis of 52 patients. Ultraschall Med. 2016:(4)37:379-85.

31. Moazedi-Fuerst FC, Zechner PM, Tripolt NJ, Kielhauser SM, Brickmann K, Scheidl S, Lutfi A, et al. Pulmonary echography in systemic sclerosis. Clin Rheumatol. 2012;31:1621-5.

32. Pinal-Fernandez I, Pallisa-Nunez E, Selva-O'Callaghan A, Castella-Fierro E, Simeon-Aznar CP, Fonollosa-Pla V, Vilardell-Tarres M. Pleural irregularity, a new ultrasound sign for the study of interstitial lung disease in systemic sclerosis and antisynthetase syndrome. Clin Exp Rheumatol. 2015;33:S136-41.

33. Moazedi-Fuerst FC, Kielhauser S, Brickmann K, Tripolt N, Meilinger M, Lufti A, Graninger W. Sonographic assessment of interstitial lung disease in patients with rheumatoid arthritis, systemic sclerosis and systemic lupus erythematosus. Clin Exp Rheumatol. 2015;33:S87-91.

34. Sperandeo M, De Cata A, Molinaro F, Trovato FM, Catalano D, Simeone A, Varriale A, et al. Ultrasound signs of pulmonary fibrosis in systemic sclerosis as timely indicators for chest computed tomography. Scand J Rheumatol. 2015;44:389-98.

35. Moazedi-Fuerst FC, Kielhauser SM, Scheidl S, Tripolt NJ, Lutfi A, Yazdani-Biuki $B$, Dejaco $C$, et al. Ultrasound screening for interstitial lung disease in rheumatoid arthritis. Clin Exp Rheumatol. 2014;32:199-203.

36. Cogliati C, Antivalle M, Torzillo D, Birocchi S, Norsa A, Bianco R, Costantino $\mathrm{G}$, et al. Standard and pocket-size lung ultrasound devices can detect interstitial lung disease in rheumatoid arthritis patients. Rheumatology (Oxford). 2014;53:1497-503.

37. Vasco PG, de Luna CG, Garrido IM, Pinilla JM, Rodriguez GF, Mateo JJ, Ruiz DC. Assessment of interstitial lung disease in Sjogren's syndrome by lung ultrasound: a pilot study of correlation with high-resolution chest tomography. Intern Emerg Med. 2017;12:327-31.

38. Pinal Fernandez I, Pallisa Nunez E, Selva-O'Callaghan A, Castella-Fierro E, Martinez-Gomez X, Vilardell-Tarres M. Correlation of ultrasound B-lines with high-resolution computed tomography in antisynthetase syndrome. Clin Exp Rheumatol. 2014;32:404-7.

39. Gargani L. Lung ultrasound: a new tool for the cardiologist. Cardiovasc Ultrasound. 2011;9:6.

40. Lichtenstein DA. Ultrasound in the management of thoracic disease. Crit Care Med. 2007;35:S250-61. 
41. Castelino FV, Varga J. Interstitial lung disease in connective tissue diseases: evolving concepts of pathogenesis and management. Arthritis Res Ther. 2010;12:213.

42. Matucci-Cerinic M, Kahaleh B, Wigley FM. Review: evidence that systemic sclerosis is a vascular disease. Arthritis Rheum. 2013;65:1953-62.

43. Brattain L, Telfer BA, Liteplo AS, Noble VE. Automated B-line scoring on thoracic sonography. J Ultrasound Med. 2013;32:2185-90.

44. Raso R, Tartarisco G, Matucci Cerinic M, Pioggia G, Picano E, Gargani L. A soft computing-based B-line analysis for objective classification of severity of pulmonary edema and fibrosis. JACC Cardiovasc Imaging. 2015;8:495-6.

45. Laria A, Lurati A, Scarpellini M. Ultrasound in rheumatologic interstitial lung disease: a case report of nonspecific interstitial pneumonia in rheumatoid arthritis. Case Rep Rheumatol. 2015;2015:107275.

46. Buda N, Kosiak W, Smolenska Z, Zdrojewski Z. Transthoracic lung ultrasound in the monitoring of interstitial lung disease: a case of scleroderma. Pol Arch Med Wewn. 2013;123:721-2.

47. Delle Sedie A, Carli L, Cioffi E, Bombardieri S, Riente L. The promising role of lung ultrasound in systemic sclerosis. Clin Rheumatol. 2012;31:1537-41.

48. Volpicelli G, Caramello V, Cardinale L, Mussa A, Bar F, Frascisco MF. Detection of sonographic B-lines in patients with normal lung or radiographic alveolar consolidation. Med Sci Monit. 2008;14:CR122-8.

49. Pingitore A, Garbella E, Piaggi P, Menicucci D, Frassi F, Lionetti V, Piarulli A, et al. Early subclinical increase in pulmonary water content in athletes performing sustained heavy exercise at sea level: ultrasound lung comet-tail evidence. Am J Physiol Heart Circ Physiol. 2011;301:H2161-7.

50. Chiesa AM, Ciccarese F, Gardelli G, Regina UM, Feletti F, Bacchi Reggiani ML, Zompatori M. Sonography of the normal lung: comparison between young and elderly subjects. J Clin Ultrasound. 2014;43:230-4. 